\title{
Metric dimensions of minor excluded graphs and minor exclusion in groups
}

\author{
Mikhail I. Ostrovskii and David Rosenthal* \\ Department of Mathematics and Computer Science \\ St. John's University \\ 8000 Utopia Parkway \\ Queens, NY 11439 \\ USA \\ Fax: (718) 990-1650 \\ e-mails: ostrovsm@stjohns.edu, rosenthd@stjohns.edu
}

October 9, 2018

\begin{abstract}
An infinite graph $\Gamma$ is minor excluded if there is a finite graph that is not a minor of $\Gamma$. We prove that minor excluded graphs have finite Assouad-Nagata dimension and study minor exclusion for Cayley graphs of finitely generated groups. Our main results and observations are: (1) minor exclusion is not a group property: it depends on the choice of generating set; (2) a group with one end has a generating set for which the Cayley graph is not minor excluded; (3) there are groups that are not minor excluded for any set of generators, like $\mathbb{Z}^{3}$; (4) minor exclusion is preserved under free products; and (5) virtually free groups are minor excluded for any choice of finite generating set.
\end{abstract}

Keywords: Assouad-Nagata dimension, Cayley graph, ends of a group, free product, graph minor.

2010 Mathematics Subject Classification. Primary: 20F65; Secondary: 05C63, 05C83, 46B85.

\section{Introduction}

A finite graph $M$ is a minor of a connected graph $\Gamma$ if there is a finite set $\left\{V_{i}\right\}$ of pairwisedisjoint finite connected subgraphs of $\Gamma$ (called branch sets) such that the set $\left\{V_{i}\right\}$ is in one-to-one correspondence with the set of vertices $\left\{v_{i}\right\}$ of $M$, and for every edge in $M$ between vertices $v_{i}$ and $v_{j}$ in $M$, there is an edge in $\Gamma$ between the corresponding branch sets $V_{i}$ and $V_{j}$. The graph $\Gamma$ is minor excluded if there is a finite graph that is not a minor

*The first-named author was supported by NSF DMS-1201269. The second-named author was supported by the Simons Foundation \#229577. The authors are very thankful to Florent Baudier, Genady Grabarnik, Volodymyr Nekrashevych, Henrik Rueping and Andreas Thom for useful discussions. 
of $\Gamma$. Since every finite graph is a subgraph of some complete graph, $\Gamma$ is minor excluded if and only if there exists a natural number $m$ such that the complete graph $K_{m}$ on $m$ vertices is not a minor of $\Gamma$. Minor exclusion of groups is about minor exclusion of Cayley graphs.

Minor exclusion plays an important role in graph theory. The well-known Kuratowski Theorem states that a finite graph is planar if and only if the complete graph on five vertices, $K_{5}$, and the complete bipartite graph on six vertices, $K_{3,3}$, are excluded as minors. If one defines an infinite graph to be planar provided there is an embedding of the graph into $\mathbb{R}^{2}$, then Kuratowski's Theorem generalizes to infinite graphs as well [8] (the theory of planarity of infinite graphs is somewhat different if the accumulation points of vertices are not allowed; see [12] and references therein). A finitely generated group $G$ is called planar if there exists a finite symmetric generating set $S$ of $G$ for which Cay $(G, S)$ is planar. There is extensive literature on planar groups (see [9, 10, 12, 19, 27, 30]), and, since planar graphs can be described in terms of minor exclusion, this study is a part of the considered subject matter. As for the study of minor exclusion for groups in general, we found only one other paper [2].

Embedding groups into Banach spaces is a very useful tool for studying groups with respect to applications in topology, most notably to the Novikov Conjecture (see, for example, 23]). It is known from the works [17] and [26] that problems about embeddability of graphs into Banach spaces are closely related to the theory of minors in graphs. Related to embedding questions is the study of various large-scale notions of dimension for groups, such as asymptotic dimension and Assouad-Nagata dimension (see [13], where many of such notions are originated, and [6]).

Our first goal in this paper is to show that if an infinite graph $\Gamma$ is minor excluded, then $\Gamma$ has finite Assouad-Nagata dimension (Theorem 2.2). This also implies that the asymptotic dimension of an infinite minor excluded graph is finite. After that we study the notion of minor exclusion for groups. Our main results and observations on minor exclusion for groups are:

- If $G$ is a finitely generated group with one end, then there is a generating set $S$ in $G$ such that Cay $(G, S)$ is not minor excluded (Theorem 3.10). Since $\mathbb{Z}^{2}$ has one end and its standard Cayley graph is planar (and thus minor excluded), this result implies that the minor exclusion of $\operatorname{Cay}(G, S)$ is not a group property, in the sense that it depends on the generating set $S$. Actually, the fact that minor exclusion is not a group property is much simpler than Theorem 3.10 (see Example 3.1).

- There is a large class of groups whose Cayley graphs are not minor excluded for any choice of a generating set (Section 3.1). This class includes all groups containing $\mathbb{Z}^{3}$ as a subgroup. Theorem 2.2 also has a corollary of this type (see Remark 2.5).

- A virtually free group is minor excluded for any choice of generating set (Theorem 3.6).

- Minor exclusion is preserved under free products (Theorem 3.8). This result generalizes a result of Arzhantseva and Cherix that planarity is preserved under free products. 


\section{Minor exclusion and metric dimensions}

An infinite graph $\Gamma$ is connected if there is a (finite) path between any two vertices of $\Gamma$. A finite graph $M$ is a minor of a connected graph $\Gamma$ if there is a finite set $\left\{V_{i}\right\}$ of pairwisedisjoint finite connected subgraphs of $\Gamma$ (called branch sets) such that the set $\left\{V_{i}\right\}$ is in one-to-one correspondence with the set of vertices $\left\{v_{i}\right\}$ of $M$, and for every edge in $M$ between vertices $v_{i}$ and $v_{j}$ in $M$, there is an edge in $\Gamma$ between the corresponding branch sets $V_{i}$ and $V_{j}$. The graph $\Gamma$ is minor excluded if and only if there exists a natural number $m$ such that the complete graph $K_{m}$ on $m$ vertices is not a minor of $\Gamma$. (Our graph theory terminology and notation mostly follows [7].)

Definition 2.1 ([3, 18]). Let $X$ be a metric space and $d \in \mathbf{N}$. The Assouad-Nagata dimension (or just Nagata dimension) of $X$ is at most $d$ if there exists a $\gamma \in(0, \infty)$ such that for every $s>0$ there exists a cover $\mathcal{U}$ of $X$ with $s$-multiplicity at most $d+1$ (i.e., each closed ball of radius $s$ in $X$ intersects at most $d+1$ elements of $\mathcal{U}$ ), whose elements each have diameter at most $\gamma \cdot s$.

Theorem 2.2. If $\Gamma$ is a connected graph with finite degrees excluding the complete graph $K_{m}$ as a minor, then $\Gamma$ has Assouad-Nagata dimension at most $4^{m}-1$.

Remark 2.3. An immediate corollary to Theorem 2.2 is that the asymptotic dimension of a minor excluded graph is finite. This is because, by definition, the asymptotic dimension of $X$ is at most $d$ if for every $s>0$ there exists a cover $\mathcal{U}$ of $X$ with $s$-multiplicity at most $d+1$, whose elements have uniformly bounded diameter. Thus, the asymptotic dimension of $X$ is bounded above by the Assouad-Nagata dimension of $X$.

Remark 2.4. In [24] it was shown that minor excluded connected infinite graphs with finite degrees of vertices admit coarse embeddings into a Hilbert space. Combining Theorem 2.2 with the results of Naor and Silberman [22] we get the following strengthening of the result of [24]: any snowflaking of a minor excluded connected graph with finite degrees admits a bilipschitz embedding into a Hilbert space (snowflaking means passing from the metric space $(X, d)$ to the metric space $\left(X, d^{\theta}\right)$, where $\left.\theta \in(0,1)\right)$.

Proof of Theorem 2.2. Observe that for graphs (considered as vertex sets with the shortest path metric, where each edge has length 1 ) it suffices to consider $s \in \mathbb{N}$ (although restricting $s$ to integers will cause us to increase $\gamma$ ). So we need to prove that there exists $0<\gamma<\infty$ (which may depend on $m$ ) such that for every $s \in \mathbb{N}$ there is a cover of $\Gamma$ with sets of diameter at most $\gamma \cdot s$ and $s$-multiplicity at most $4^{m}$. To find such a cover we construct $4^{m}$ "partitions" of $\Gamma$, following the approach of [17] with a slight modification. Elements of the partitions will be defined as connected components of the graph obtained from $\Gamma$ after removing $m$ sets of edges $\left\{F_{i}\right\}_{i=1}^{m}$, constructed as follows.

Enumerate all of the vertices of $\Gamma$ as $\left\{v_{1}, v_{2}, \ldots\right\}$ (it is clear that connected graphs with finite degrees are countable), and let $R=s+3$. Let $\delta_{1}$ be one of the elements of the set $\{0, R, 2 R, 3 R\}$ and define $F_{1}$ to be the set of all edges that join vertices $u$ satisfying $d\left(u, v_{1}\right)=4 R j+\delta_{1}$, for some $j \in \mathbb{N}$, to vertices $w$ satisfying $d\left(w, v_{1}\right)=4 R j+\delta_{1}+1$ for the same $j$. Now delete $F_{1}$ from the edge set of $\Gamma$. It is clear that unless $d\left(u, v_{1}\right) \leq 4 R+\delta_{1}$ for all $u \in V(\Gamma)$, we get a disconnected graph. To construct $F_{2}$, pick $\delta_{2} \in\{0, R, 2 R, 3 R\}$ 
independently from the choice of $\delta_{1}$. In each component $C$ of $\Gamma \backslash F_{1}$ choose a vertex $v_{i(C)}$ with smallest index $i(C)$ over all vertices of $C$. Consider the set of all edges that join vertices $u$ satisfying $d_{C}\left(u, v_{i(C)}\right)=4 R j+\delta_{2}$, for some $j \in \mathbb{N}$, to vertices $w$ satisfying $d_{C}\left(w, v_{i(C)}\right)=4 R j+\delta_{2}+1$, where $d_{C}$ is the path-length metric (or shortest path distance) of $C$. Define $F_{2}$ as the union of such edge sets over all components $C$ of the graph $\Gamma \backslash F_{1}$ obtained from $\Gamma$ after the deletion of edges of $F_{1}$. To construct $F_{3}$, pick $\delta_{3} \in\{0, R, 2 R, 3 R\}$ independently from the choice of $\delta_{1}$ and $\delta_{2}$. Repeat the procedure from the previous paragraph for each component of the graph obtained from $\Gamma$ after the removal of $F_{1} \cup F_{2}$. Continue in the obvious way, making a series of $m$ cuts of this type. We call the vertex sets of the connected components of $\Gamma \backslash \cup_{i=1}^{m} F_{i}$ clusters; this set of clusters yields a partition $\mathcal{P}$ of $\Gamma$. Construct such partitions for all possible choices of $\delta_{1}, \ldots, \delta_{m}$. We get $4^{m}$ different partitions $\left\{\mathcal{P}_{p}\right\}_{p=1}^{4^{m}}$ of $\Gamma$.

Define a cover $\mathcal{U}$ of $\Gamma$ as follows. For each cluster $T$ in some partition $\mathcal{P}_{p}$, let $U_{T}$ be the set of all vertices of $T$ satisfying the following conditions: (1) they have distance at least $s+1$ from the ends of the edges of $F_{1}$ in the original distance of $\Gamma$; and (2) for each integer $k, 2 \leq k \leq m$, they have distance at least $s+1$ from the ends of edges of $F_{k}$ in the shortest-path metric for the graph $\Gamma \backslash \cup_{i=1}^{k-1} F_{i}$.

The set $\mathcal{U}=\left\{U_{T}\right\}$, over all clusters $T$ of all partitions $\mathcal{P}_{p}$, must form a cover. To verify this, let $w$ be a vertex in $\Gamma$. We choose $\delta_{1}, \ldots, \delta_{m}$ so that $w$ will be contained in $U_{T}$, where $T$ is the cluster of the partition corresponding to $\delta_{1}, \ldots, \delta_{m}$ that contains $w$. This can be done in the following way: select $\delta_{1} \in\{0, R, 2 R, 3 R\}$ so that $d_{\Gamma}\left(w, v_{1}\right)-\delta_{1}$ modulo $4 R$ is between $R$ and $3 R$. After removing the corresponding $F_{1}$, the vertex $w$ lands in one of the components, $C$, of the graph $\Gamma \backslash F_{1}$. Let $v_{i(C)}$ be the vertex of this component with smallest index. Select $\delta_{2} \in\{0, R, 2 R, 3 R\}$ so that $d_{C}\left(w, v_{i(C)}\right)-\delta_{2}$ modulo $4 R$ is between $R$ and $3 R$. Continue in the obvious way. It is straightforward to check, since $R=s+3$, that $w$ satisfies conditions (1) and (2) above for the obtained $F_{1}, \ldots, F_{m}$.

Klein, Plotkin, and Rao [17] proved that the assumption that $\Gamma$ does not have $K_{m}$ as a minor implies that the diameter of any cluster in any $\mathcal{P}_{p}$ is at most $\alpha(m) \cdot R$, where $\alpha(m) \in(0, \infty)$ depends only on $m$. Therefore, every element of $\mathcal{U}$ will have diameter at most $\gamma \cdot s$ if we choose $\gamma=4 \cdot \alpha(m)$.

It remains to show that the $s$-multiplicity of $\mathcal{U}$ is at most $4^{m}$. Since each $x$ is inside at most $4^{m}$ different clusters $T$ (one cluster from each partition), this would follow from the claim that for a given element $U_{T}$ of the cover, the ball $B(x, s)$ can intersect $U_{T}$ only if $x \in T$. To prove this claim, it suffices to establish the following statement by induction on $k$ :

If $x$ is separated from $T$ by the edge cut $\cup_{i=1}^{k} F_{i}$, but not by $\cup_{i=1}^{k-1} F_{i}$ (the latter set is assumed to be empty for $k=1$ ), then $x$ has distance at least $s$ from $U_{T}$.

For $k=1$ this is obvious, by item (1) in the definition of $U_{T}$. Let $k=2$. Assume that there is a path of length at most $s$ joining $x$ and $U_{T}$. If this path does not use any edges of $F_{1}$, then the path-length distance in $\Gamma \backslash F_{1}$ between $x$ and $U_{T}$ is also at most $s$. But this contradicts item (2) in the definition of $U_{T}$. If this path uses an edge of $F_{1}$, we get a contradiction with what we proved for $k=1$. The inductive step is the same as in the proof of the case $k=2$.

Remark 2.5. See [23] for the definition of "compression". Remark 2.4 shows that Theo- 
rem 2.2 in combination with the results of Naor and Silberman [22] implies that minor excluded groups have compression 1. Therefore, the Cayley graph of a group that does not have compression 1 is not minor excluded with respect to an arbitrary set of generators.

Remark 2.6. It is worth mentioning that estimates from [17 for $\alpha(r)$ were improved in [11] (see also a presentation of results of [11, [17, and [26] in [25, Section 3.2]).

\section{Minor exclusion for groups}

Let $G$ be a finitely generated group, and let $S$ be a finite generating set for $G$. Throughout this paper, we assume that $S$ does not contain the identity element of $G$ and that $S$ is symmetric, i.e., $s \in S$ if and only if $s^{-1} \in S$. To study minor exclusion of $G$, we will always use the right-invariant Cayley $\operatorname{graph}$, Cay $(G, S)$, i.e., the graph with vertex set $G$ and edge set defined by the condition: $u v$ is an edge between $u, v \in G$ if and only if $u=s v$ for some $s \in S$. The group $G$ acts on the right of this graph. (Our references for group theory are 21] and [28].)

When considering minor exclusion for groups, one quickly realizes, as Example 3.1 shows, that minor exclusion is not a group property. That is, it depends not only on the group, but also on the choice of a generating set.

Example 3.1. The Cayley graph $\operatorname{Cay}\left(\mathbb{Z}^{2}, S_{1}\right)$, where $S_{1}=\{( \pm 1,0),(0, \pm 1)\}$, is minor excluded by the well-known Kuratowski theorem, since it is a planar graph. On the other hand, the Cayley graph of $\mathbb{Z}^{2}$ with respect to the, slightly bigger, set of generators $S_{2}=$ $\{( \pm 1,0),( \pm 2,0),(0, \pm 1)\}$, is not minor excluded.

Proof. Let $m \in \mathbb{N}$ be given. Construct branch sets of a $K_{m}$-minor in $\operatorname{Cay}\left(\mathbb{Z}^{2}, S_{2}\right)$ as follows:

$$
\begin{gathered}
V_{1}=\{(2,1),(\text { even }, 1),(2 m, 1)\} \\
V_{2}=\left\{(3,1),(3,2),(4,2),\left({ }^{\text {even }, 2)},(2 m, 2)\right\}\right. \\
\vdots \\
V_{k}=\left\{(2 k-1,1),(2 k-1,2),(2 k-1,3), \ldots,(2 k-1, k),(2 k, k),{ }^{(\text {even }, k)},(2 m, k)\right\} \\
\vdots \\
V_{m}=\{(2 m-1,1), \ldots,(2 m-1, m),(2 m, m)\} .
\end{gathered}
$$

It is straightforward to check that each $V_{k}$ is the vertex set of a connected subgraph of Cay $\left(\mathbb{Z}^{2}, S_{2}\right)$ and that any two of these vertex sets are joined by an edge.

\subsection{Groups that are not minor excluded for any choice of generators}

It is interesting that if the group $\mathbb{Z}^{2}$ is increased even "slightly", then we get a group that is not minor excluded for any set of generators. We mean the following result.

Lemma 3.2. Let $C$ be a nontrivial cyclic group. Then $\mathbb{Z}^{2} \times C$ is not minor excluded for any set of generators. 
Proof. Let $S$ be a finite symmetric generating set for $\mathbb{Z}^{2} \times C$. Since $\mathbb{Z}^{2} \times C$ is abelian, there are distinct generators $s_{1}, s_{2}, s_{3} \in S$ such that the subgroup $\left\langle s_{1}, s_{2}\right\rangle$ generated by $s_{1}$ and $s_{2}$ is isomorphic to $\mathbb{Z}^{2}$ and $s_{3} \notin\left\langle s_{1}, s_{2}\right\rangle$. Let $m \in \mathbb{N}$ be given and construct branch sets of Cay $\left(\mathbb{Z}^{2} \times C, S\right)$ as follows:

$$
\begin{gathered}
V_{1}=\left\{s_{1}, 2 s_{1}, 3 s_{1}, \ldots, m s_{1}\right\} \\
V_{2}=\left\{2 s_{1}+s_{3}, 2 s_{1}+s_{2}+s_{3}, 2 s_{1}+s_{2}, \ldots, m s_{1}+s_{2}\right\} \\
\vdots \\
V_{k}=\left\{k s_{1}+j s_{2}+s_{3}: 0 \leq j \leq k-1\right\} \cup\left\{l s_{1}+(k-1) s_{2}: k \leq l \leq m\right\} \\
\vdots \\
V_{m}=\left\{m s_{1}+s_{3}, m s_{1}+s_{2}+s_{3}, \ldots, m s_{1}+(m-1) s_{2}+s_{3}, m s_{1}+(m-1) s_{2}\right\} .
\end{gathered}
$$

It is straightforward to check that each $V_{k}$ is the vertex set of a connected subgraph of $\operatorname{Cay}\left(\mathbb{Z}^{2} \times C, S\right)$ and that any two of these vertex sets are joined by an edge. Therefore, we have constructed a $K_{m}$ minor in $\operatorname{Cay}\left(\mathbb{Z}^{2} \times C, S\right)$ for every $m \in \mathbb{N}$; that is, Cay $\left(\mathbb{Z}^{2} \times C, S\right)$ is not minor excluded.

Remark 3.3. The group $\mathbb{Z}^{2} \times \mathbb{Z}_{2}$ is in a sense the smallest group satisfying the assumptions of Lemma 3.2. It is worth noting that when $C$ is a finite group, $\mathbb{Z}^{2} \times C$ is quasi-isometric to $\mathbb{Z}^{2}$ (see [5, p. 138] for the definition).

The following is a generalization of Babai's result that every subgroup of a planar group is planar [4].

Theorem 3.4. Let $G$ be a finitely generated group containing a finitely generated subgroup $H$. If $H$ is not minor excluded for any set of generators, then $G$ is not minor excluded for any set of generators.

Proof. Let $X=\operatorname{Cay}(G, S)$, where $S$ is a finite symmetric generating set for $G$. We must show that there is a $K_{m}$-minor of $X$ for every $m \in \mathbb{N}$.

We begin by recalling Babai's construction of a Cayley graph $X^{\prime}$ for the subgroup $H$ [4]. There is a connected subgraph $T$ of $X$ that contains precisely one vertex for each $H$-orbit in $V(X)$. This means that if $h \in H \backslash\{e\}$, then $V(T \cdot h) \cap V(T)=\emptyset$ and $\bigcup_{h \in H} V(T \cdot h)=V(X)$. Then $X^{\prime}$ is obtained by collapsing each $T \cdot h$. That is, the vertex set of $X^{\prime}$ is $V\left(X^{\prime}\right)=\{T \cdot h: h \in H\}$ and there is one edge in $X^{\prime}$ between the vertices $T \cdot h$ and $T \cdot h^{\prime}$ if there is an edge in $X$ connecting the subsets $T \cdot h$ and $T \cdot h^{\prime}$. Thus, $X^{\prime}$ is connected (since $X$ is connected), $H$ acts on $X^{\prime}$, and the $H$-action is free and transitive on $V\left(X^{\prime}\right)$. Therefore, $X^{\prime}$ is a Cayley graph for $H$.

The generating set for $H$ corresponding to $X^{\prime}$ is the set $S^{\prime}$ of all $k \in H$ such that there is an edge in $X^{\prime}$ between $T$ and $T \cdot k$. Note that $S^{\prime}$ might be infinite. Since $H$ is finitely generated, however, there must be a finite subset $S^{\prime \prime} \subset S^{\prime}$ that generates $H$. Under the identification of $X^{\prime}$ with Cay $\left(H, S^{\prime}\right)$, let $X^{\prime \prime}$ be the subgraph of $X^{\prime}$ corresponding to the subgraph $\operatorname{Cay}\left(H, S^{\prime \prime}\right) \subset \operatorname{Cay}\left(H, S^{\prime}\right)$. By assumption, $H$ is not minor excluded with respect to $S^{\prime \prime}$. Thus, for every $m \in \mathbb{N}$, there is a $K_{m}$-minor of $X^{\prime \prime}$. It is clear from the construction that a $K_{m}$-minor of $X^{\prime \prime}$ yields a $K_{m}$-minor of $X$. In other words, $G$ is not minor excluded with respect to $S$. 
By Lemma 3.2, we have the following corollary to Theorem 3.4.

Corollary 3.5. Let $G$ be a finitely generated group that contains $\mathbb{Z}^{2} \times C$ as a subgroup, where $C$ is a nontrivial cyclic group. Then the Cayley graph of $G$ is not minor excluded for any set of generators.

\subsection{Minor exclusion for groups containing finitely generated free sub- groups of finite index}

Next we consider groups that contain a finitely generated free group as a subgroup of finite index. Let $\mathbb{F}_{n}$ denote the free group on $n$ generators.

Theorem 3.6. Let $G$ be a group containing $\mathbb{F}_{n}$ as a subgroup of finite index and let $S$ be a finite symmetric generating set for $G$. Then $\operatorname{Cay}(G, S)$ is minor excluded.

Proof. Let $\left\{x_{1}, \ldots, x_{n}\right\} \subset G$ be the basis of $\mathbb{F}_{n}$, and let $\left\{g_{1}=1, g_{2}, \ldots, g_{k}\right\}$ be representatives of the left cosets of $\mathbb{F}_{n}$ in $G$. As a set, $G$ may be identified with the Cartesian product $G=\left\{g_{1}, \ldots, g_{k}\right\} \times \mathbb{F}_{n}$ since each $g \in G$ can be uniquely represented as $g=g_{i} f$, where $i \in\{1, \ldots, k\}$ and $f \in \mathbb{F}_{n}$. Let $O=\left\{x_{1}, \ldots, x_{n}, x_{1}^{-1}, \ldots, x_{n}^{-1}\right\}$ and let $\operatorname{Cay}\left(\mathbb{F}_{n}, O\right)$ be the corresponding Cayley graph; denote the associated distance function for this graph by $d_{\mathbb{F}_{n}}$. To prove the theorem, assume the contrary. That is, assume that the graph Cay $(G, S)$ has a $K_{m}$-minor for every $m \in \mathbb{N}$.

Lemma 3.7. Let $e$ be an edge in $\operatorname{Cay}\left(\mathbb{F}_{n}, O\right)$ and let $A(e)$ and $B(e)$ be the vertex sets of the connected components of $\operatorname{Cay}\left(\mathbb{F}_{n}, O\right) \backslash\{e\}$. The number of edges in Cay $(G, S)$ connecting $\left\{g_{1}, \ldots, g_{k}\right\} \times A(e)$ and $\left\{g_{1}, \ldots, g_{k}\right\} \times B(e)$ is finite and bounded from above independently of the choice of $e$.

Proof. The lemma is an immediate consequence of the following claim. If $u, v \in G$ are adjacent in $\operatorname{Cay}(G, S)$, and $u=g_{i(1)} f_{1}$ and $v=g_{i(2)} f_{2}$, where $i(1), i(2) \in\{1, \ldots, k\}$ and $f_{1}, f_{2} \in \mathbb{F}_{n}$, then $d_{\mathbb{F}_{n}}\left(f_{1}, f_{2}\right) \leq M$, where $M \in \mathbb{N}$ depends on $G, S$, and the choice of $\left\{g_{1}, \ldots, g_{k}\right\}$ (but not on the choice of $u$ and $v$ ). To verify this claim, note that there is an $s \in S$ such that $g_{i(2)} f_{2}=s g_{i(1)} f_{1}$, since $u$ and $v$ are adjacent in Cay $(G, S)$. Also, for each $i \in\{1, \ldots, k\}$ and $s \in S$, we have $s g_{i}=g_{j(i, s)} f(i, s)$, for some $j(i, s) \in\{1, \ldots, k\}$ and $f(i, s) \in \mathbb{F}_{n}$. This implies that $f_{2}=f(i(1), s) f_{1}$. Therefore, $d_{\mathbb{F}_{n}}\left(f_{1}, f_{2}\right) \leq \max _{s \in S} \max _{i \in\{1, \ldots, k\}} d_{\mathbb{F}_{n}}(f(i, s), 1)$. This maximum is over a finite set, so the claim follows.

Let $D$ denote the upper bound obtained in Lemma 3.7. Given a $K_{m}$-minor in $\operatorname{Cay}(G, S)$, the number of edges of $\operatorname{Cay}(G, S)$ connecting $\left\{g_{1}, \ldots, g_{k}\right\} \times A(e)$ and $\left\{g_{1}, \ldots, g_{k}\right\} \times B(e)$ must be at least $R(e)+k_{A}(e) \cdot k_{B}(e)$, where $R(e)$ is the number of branch sets of the $K_{m}$-minor intersecting both of the sets (such branch sets are said to cross e), $k_{A}(e)$ is the number of branch sets that are completely contained in $\left\{g_{1}, \ldots, g_{k}\right\} \times A(e)$, and $k_{B}(e)$ is the number of branch sets completely contained in $\left\{g_{1}, \ldots, g_{k}\right\} \times B(e)$. We will show that, if $m$ is large enough, the inequality $D \geq R(e)+k_{A}(e) \cdot k_{B}(e)$ leads to a contradiction for a suitably chosen edge $e$.

Choose $m>\max \{6 n D, 3 k\}$. Then the following two conditions are satisfied: 
(1) $\frac{1}{2 n-1}\left(\frac{2 m}{3}-\frac{m}{6 n}-k\right)>\frac{m}{6 n}$;

(2) $\frac{m}{6 n}>D$.

By condition (2), for any edge $e$ in $\operatorname{Cay}\left(\mathbb{F}_{n}, O\right)$ the number of branch sets, $R(e)$, that cross both $\left\{g_{1}, \ldots, g_{k}\right\} \times A(e)$ and $\left\{g_{1}, \ldots, g_{k}\right\} \times B(e)$ is less than $\frac{m}{6 n}$. Therefore, because of condition (2), we can establish a contradiction with the inequality $D \geq R(e)+k_{A}(e) \cdot k_{B}(e)$ if we find an edge for which $k_{A}(e) \cdot k_{B}(e) \geq \frac{m}{6 n}$. We do this by showing that there exists an edge for which both $k_{A}(e)$ and $k_{B}(e)$ are positive and one of them is greater than $\frac{m}{6 n}$.

Pick an edge $e_{0}$ in $\operatorname{Cay}\left(\mathbb{F}_{n}, O\right)$ and assume that $k_{A}\left(e_{0}\right) \geq k_{B}\left(e_{0}\right)$. If $k_{B}\left(e_{0}\right) \geq \frac{m}{6 n}$, then we are done. So assume that $k_{B}\left(e_{0}\right)<\frac{m}{6 n}$. Let $e_{1}, \ldots, e_{2 n-1}$ be the edges that have a common vertex, $f$, with $e_{0}$ and are contained in $\left\{g_{1}, \ldots, g_{k}\right\} \times A\left(e_{0}\right)$. Let $A\left(e_{1}\right), \ldots, A\left(e_{2 n-1}\right)$ denote the vertex sets of the components of $\operatorname{Cay}\left(\mathbb{F}_{n}, O\right) \backslash\left\{e_{1}\right\}, \ldots, \operatorname{Cay}\left(\mathbb{F}_{n}, O\right) \backslash\left\{e_{2 n-1}\right\}$, respectively, that do not contain $e_{0}$. As noted above, $R\left(e_{i}\right)<\frac{m}{6 n}$ for every $i$. That is, each of $e_{0}, e_{1}, \ldots, e_{2 n-1}$ is crossed by fewer than $\frac{m}{6 n}$ branch sets. Thus, there are more than $\frac{2 m}{3}$ branch sets that do not cross any of the edges $e_{0}, e_{1}, \ldots, e_{2 n-1}$. On the other hand, since there are at most $k$ branch sets in the set $\left\{g_{1}, \ldots, g_{k}\right\} \times\{f\}$, the number of branch sets that do not cross any of the edges $e_{0}, e_{1}, \ldots, e_{2 n-1}$ is at most

$$
\left(\sum_{i=1}^{2 n-1} k_{A}\left(e_{i}\right)\right)+k_{B}\left(e_{0}\right)+k<\left(\sum_{i=1}^{2 n-1} k_{A}\left(e_{i}\right)\right)+\frac{m}{6 n}+k .
$$

Therefore, $\left(\sum_{i=1}^{2 n-1} k_{A}\left(e_{i}\right)\right)+\frac{m}{6 n}+k>\frac{2 m}{3}$. Applying (1), this implies that at least one of the sets $\left\{g_{1}, \ldots, g_{k}\right\} \times A\left(e_{1}\right), \ldots,\left\{g_{1}, \ldots, g_{k}\right\} \times A\left(e_{2 n-1}\right)$ contains more than $\frac{m}{6 n}$ branch sets. That is, $k_{A}\left(e_{i}\right)>\frac{m}{6 n}$ for some $i, 1 \leq i \leq 2 n-1$. If $k_{B}\left(e_{i}\right)>0$, then we are done. If $k_{B}\left(e_{i}\right)=0$, then $k_{B}\left(e_{i}\right)<\frac{m}{6 n}$ and we can repeat the argument. But the argument cannot be repeated infinitely many times, since the branch sets are finite and there are only finitely many of them. Therefore, we eventually find an edge $e$ with $k_{A}(e)>\frac{m}{6 n}$ and $k_{B}(e)>0$, as claimed.

\subsection{Stability of minor exclusion with respect to free products}

Recall that a cut-vertex in a graph is a vertex whose deletion increases the number of components. The following theorem is a generalization of [2, Theorem 3(i)], which states that $\operatorname{Cay}(G * H, S \cup T)$ is planar if $\operatorname{both} \operatorname{Cay}(G, S)$ and $\operatorname{Cay}(H, T)$ are planar.

Theorem 3.8. Let $G$ and $H$ be finitely generated groups with generating sets $S$ and $T$, respectively. Let $M$ be a finite connected graph that does not contain cut-vertices. If $M$ is a minor of Cay $(G * H, S \cup T)$, then $M$ is a minor of either Cay $(G, S)$ or $\operatorname{Cay}(H, T)$.

Proof of Theorem 3.8. Let $V_{1}, \ldots, V_{m}$ be branch sets of an $M$-minor in $\operatorname{Cay}(G * H, S \cup$ $T)$. Recall that each element of $G * H$ is represented by a unique reduced word and nontrivial reduced words are products of the form $x_{n} \cdots x_{1}$, where $x_{n}, \ldots, x_{1}$ are nonidentity elements alternating between $G$ and $H$ and $x_{1}$ can be either in $G$ or in $H$ (see [28, p. 169] if needed). Recall that two vertices $u, v$ in Cay $(G * H, S \cup T)$ are adjacent if and only if $u=s v$ for some $s \in S \cup T$. This immediately implies the following observation. 
Observation 3.9. Let $x_{n} \cdots x_{1}$ and $y_{t} \cdots y_{1}$ be reduced words in Cay $(G * H, S \cup T)$ and let $x_{k-1} \cdots x_{1}=y_{k-1} \cdots y_{1}$ denote their largest common word, which can be empty. Then each path connecting $x_{n} \cdots x_{1}$ and $y_{t} \cdots y_{1}$ passes through $x_{k} \cdots x_{1}$ if $n \geq k$, and through $y_{k} \cdots y_{1}$ if $t \geq k$. If $x_{1} \in G$ and $y_{1} \in H$, or vise versa, then the path passes through 1 .

We shall use the notation $H x_{n} \cdots x_{1}=\left\{x_{n+1} \cdot x_{n} \cdots x_{1}: x_{n+1} \in H\right\}$, where $x_{n}, x_{n-2}, \ldots$ are fixed elements of $G$ and $x_{n-1}, x_{n-3}, \ldots$ are fixed elements of $H$. Similarly, we define $G y_{m} \cdots y_{1}$. Combining our assumptions with Menger's theorem [7, Section 3.3], it follows that there exist two vertices $u_{1} \in V_{1}$ and $u_{2} \in V_{2}$ such that there are two disjoint $u_{1} u_{2^{-}}$ paths in Cay $(G * H, S \cup T)$. Using Observation 3.9, we get that $u_{1}$ and $u_{2}$ are either in the same set of the form $G y_{r} \cdots y_{1}$, or in the same set of the form $H x_{m} \cdots x_{1}$. Without loss of generality, we can assume that $u_{1}$ and $u_{2}$ are in $G y_{r} \cdots y_{1}$. Since Cay $(G * H, S \cup T)$ is right-invariant, multiplying all elements of $G * H$ on the right by $\left(y_{r} \cdots y_{1}\right)^{-1}$, we may assume that $u_{1}$ and $u_{2}$ are in $G$. We claim that $V_{1} \cap G, \ldots, V_{m} \cap G$ are the branch sets of an $M$-minor in $\operatorname{Cay}(G, S)$.

The assumption that $M$ does not have cut-vertices implies that for each $i, 3 \leq i \leq m$, there is a vertex $u_{i} \in V_{i}$ for which there are two disjoint paths, one that is a $u_{1} u_{i}$-path and one that is a $u_{2} u_{i}$-path (this follows from [7, Corollary 3.3.3] with $a=v_{i}$ and $B=\left\{v_{1}, v_{2}\right\}$, where $\left\{v_{i}\right\}$ consists of vertices of $M$ corresponding to the branch sets $\left.V_{1}, \ldots, V_{m}\right)$. On the other hand, Observation 3.9 implies that two paths from different vertices of $G$ to a vertex not in $G$ cannot be disjoint. Therefore, $V_{i} \cap G \neq \emptyset$, for every $i$. Next, notice that for any two elements in $u, v \in V_{i} \cap G$ there is a uv-path $P$ in Cay $(G * H, S \cup T)$, all of whose vertices are contained in $V_{i}$. Since a path does not pass through any elements repeatedly, $P$ cannot leave $G$. Thus, the sets $V_{i} \cap G$ are connected in Cay $(G, S)$ for each $i$.

To complete the proof, we show that if there is an edge in $M$ joining the vertices corresponding to $V_{i}$ and $V_{j}$, then there is an edge in $\operatorname{Cay}(G, S)$ joining $V_{i} \cap G$ and $V_{j} \cap G$. Since $V_{1}, \ldots, V_{m}$ are branch sets of an $M$-minor, there is an edge joining $V_{i}$ and $V_{j}$. This edge must be in $\operatorname{Cay}(G, S)$, because otherwise we can find a path in $\operatorname{Cay}(G * H, S \cup T)$ that leaves $G$ through one element of $G$ and returns through another element of $G$, which is clearly impossible.

\subsection{Groups with one end}

Let $\Gamma$ be a connected, locally finite graph, and denote by $B(n, O)$ the ball of radius $n$ in $\Gamma$ centered at some fixed vertex $O$ in $\Gamma$. The number of ends in $\Gamma$ is the limit of the number of unbounded connected components in $\Gamma \backslash B(n, O)$ as $n \rightarrow \infty$. This limit exists either as a nonnegative integer or as $\infty$. (See [5, pp. 144-148] or [21, Sections 11.4-11.6] for an introduction to the theory of ends.) A basic result is that the number of ends of a Cayley graph of a finitely generated group does not depend on the choice of generating set (see [21, Theorem 11.23]).

The following theorem is the main result of this section.

Theorem 3.10. Let $G$ be a finitely generated group with one end. Then there is a finite set of generators $S$ of $G$ such that $\operatorname{Cay}(G, S)$ is not minor excluded.

Lemma 3.11. Assume that a one-ended group $G$ with finite generating set $S_{0}$ has the property that for each $m \in \mathbb{N}$ the Cayley graph Cay $\left(G, S_{0}\right)$ contains a collection of $m$ 
disjoint infinite rays. Then $G$ contains a finite generating set $S$ such that $\operatorname{Cay}(G, S)$ is not minor excluded.

Proof. Let $\left\{V_{i}\right\}_{i=1}^{m}$ be disjoint rays in $\operatorname{Cay}\left(G, S_{0}\right)$. The sets $V_{i}$, after some small modifications, will become branch sets of a $K_{m}$-minor in Cay $(G, S)$, where $S=S_{0} \cup S_{0} S_{0} \cup S_{0} S_{0} S_{0}$. (Here, $S_{0} S_{0}$ and $S_{0} S_{0} S_{0}$ are the sets of products of all pairs and triples, respectively, of elements in $S_{0}$.) That is, we will modify $\left\{V_{i}\right\}$ in such a way that the modified sets are still disjoint, each modified $V_{i}$ is connected in $\operatorname{Cay}(G, S)$, and for each $i \neq j$ there is an edge in $\operatorname{Cay}(G, S)$ joining a vertex of the modified $V_{i}$ with a vertex of the modified $V_{j}$. This will be achieved in $\frac{m(m-1)}{2}$ steps. Intuitively speaking, each step will create the desired "connection" between $V_{i}$ and $V_{j}$ for the pair $(i, j), i \neq j$.

Start with the pair $(1,2)$. Since the graph $\operatorname{Cay}(G, S)$ is connected, there is a path $P$ connecting some vertex of $V_{1}$ to some vertex of $V_{2}$. Suppose $P$ intersects another $V_{k}$. We describe a procedure for "removing" this intersection. Write $V_{k}=\left\{y_{(k, 1)}, y_{(k, 2)}, \ldots\right\}$, where the indexing starts with the ray's origin and moves out towards "infinity". The intersection of $P$ and $V_{k}$ has a first vertex and a last vertex (with respect to the indexing of the elements of $\left.V_{k}\right)$. Denote these vertices $y_{(k, s)}$ and $y_{(k, t)}(s \leq t)$, respectively.

If $s=t$, that is, if $P$ intersects $V_{k}$ in just one vertex, then replace $V_{k}$ with $V_{k}^{\prime}=$ $V_{k} \backslash\left\{y_{(k, s)}\right\}$ and leave $P$ unchanged. If $t=s+1$, replace $V_{k}$ with $V_{k}^{\prime}=V_{k} \backslash\left\{y_{(k, s)}, y_{(k, t)}\right\}$ and leave $P$ unchanged. Then, in both cases, $V_{k}^{\prime}$ is disjoint from $V_{1}$ and $V_{2}, V_{k}^{\prime}$ is connected in Cay $(G, S)$ (since $S_{0} S_{0}$ and $S_{0} S_{0} S_{0}$ are in $S$ ), and $V_{k}^{\prime}$ does not intersect $P$.

If $s+1<t$, then we modify both $P$ and $V_{k}$, as follows. Clearly we can find two paths, $L_{1}$ and $L_{2}$, using elements of $S_{0} S_{0}$ and $S_{0} S_{0} S_{0}$ such that $L_{1}$ is a path from $y_{(k, s)}$ to $y_{(k, t)}$, $L_{2}$ is a path from $y_{(k, s+1)}$ to $y_{(k, t+1)}, L_{1}$ and $L_{2}$ belong to $V_{k}$, and $L_{1}$ is disjoint from $L_{2}$. Now we replace the piece of $P$ that connects $y_{(k, s)}$ to $y_{(k, t)}$ with $L_{1}$ to obtain a new path $P^{\prime}$, and we create $V_{k}^{\prime}$ from $V_{k}$ by removing all the vertices in $V_{k}$ from $y_{(k, s)}$ to $y_{(k, t)}$ and adding the vertices of $L_{2}$, i.e., $V_{k}^{\prime}=\left(V_{k} \backslash\left\{y_{(k, i)}: s \leq i \leq t\right\}\right) \cup L_{2}$. Then, $V_{k}^{\prime}$ is disjoint from $V_{1}$ and $V_{2}, V_{k}^{\prime}$ is connected in $\operatorname{Cay}(G, S)$, and $V_{k}^{\prime}$ does not intersect $P^{\prime}$.

The procedure used for "removing" the intersection of $P$ with $V_{k}$ did not introduce any new intersections, although it is possible that some other intersections disappeared in the process. (Since the sets $\left\{V_{i}\right\}$ are disjoint, $P$ cannot intersect more than one $V_{k}$ in the same place.) Thus, the procedure can be repeated to "remove" each intersection one at a time until we obtain a path from $V_{1}$ to $V_{2}$ that does not intersect any of the modified $V_{i}$ 's.

We continue to use the method described above for constructing the desired connection between $V_{1}$ and $V_{2}$ until we establish "connections" for every pair $(i, j), i \neq j$. Specifically, once we have established "connections" between some of the $V_{i}$ and would then like to arrange a new connection, we remove a ball, $B$, centered at 1 from $\operatorname{Cay}(G, S)$, whose radius is large enough to contain all vertices that were used for previous paths and all pieces of $V_{k}$ 's that were involved in the previous modifications. Since $G$ has one end, Cay $(G, S) \backslash B$ has an unbounded connected component, $\Theta$. Thus, we can use the method above on the infinite connected pieces of the rays $V_{i}$ in $\Theta$. The new modifications will not destroy previous connections because they are made away from the previously constructed connections. After "connections" have been constructed for all pairs $(i, j), i \neq j$, the sets obtained from the final modification will be branch sets for $K_{m}$ in $\operatorname{Cay}(G, S)$.

Proof of Theorem 3.10. By Lemma 3.11, it suffices to construct, for an arbitrary $m \in \mathbb{N}$, 
a collection of $m$ disjoint rays in $\operatorname{Cay}\left(G, S_{0}\right)$, where $S_{0} \subset G$ is some finite generating set. We construct such rays using Menger's theorem [7, Section 3.3], which states: if vertex sets $A$ and $B$ in a graph cannot be separated by removing fewer than $k$ vertices, then there are $k$ disjoint paths joining $A$ and $B$. In [7] this result is proved for finite graphs, but it also holds for infinite locally finite graphs (and even in a more general context; see [1] and [14]).

Begin by fixing a finite generating set $\widetilde{S}$ in $G$. For each vertex $v$, let $\ell(v)$ denote the length of $v$, i.e., the distance from $v$ to the unit element 1 in $\operatorname{Cay}(G, \widetilde{S})$. Consider the following alternatives.

(i) There is some $m \in \mathbb{N}$ such that for each $R \in \mathbb{N}$ there is a set $C_{R}$, consisting of $m$ vertices of length at least $R$, such that the removal of $C_{R}$ from $\operatorname{Cay}(G, \widetilde{S})$ disconnects 1 from the infinite component.

(ii) There is no such $m$.

Consider case (ii). Let $m \in \mathbb{N}$ be given and choose $S_{0}=\widetilde{S}$. Then there is an $R \in \mathbb{Z}$ such that the removal of $m$ vertices of length at least $R$ in Cay $\left(G, S_{0}\right)$ cannot disconnect 1 and "infinity". Let $L$ be a natural number bigger than $R$. Use Menger's theorem on a one-element set $\left\{w_{L}\right\}$ with $\ell\left(w_{L}\right)=L>R$ and an $m$-element subset $A$ of vertices from $\{v: \ell(v)=R\}$. Such a subset exists since, by assumption, $\#\{v: \quad \ell(v)=R\}>m$ (otherwise this set would be an $m$-element set disconnecting 1 and "infinity"). This yields a sequence $\left\{\left(P_{1}^{L}, \ldots, P_{m}^{L}\right)\right\}_{L=R+1}^{\infty}$ of $m$-tuples of disjoint paths joining $A$ and $w_{L}$. Since $\operatorname{Cay}\left(G, S_{0}\right)$ is locally finite, we can find a convergent subsequence in $\left\{P_{1}^{L}\right\}_{L=R+1}^{\infty}$. Let $I_{1}$ be the corresponding set of indices and $P_{1}$ be the limiting path. Consider the sequence $\left\{P_{2}^{L}\right\}_{L \in I_{1}}$. It contains a convergent subsequence. It is easy to check that the limit $P_{2}$ of this subsequence is disjoint from $P_{1}$. Continue in the obvious way to obtain $m$ disjoint rays in $\operatorname{Cay}\left(G, S_{0}\right)$.

Now consider case (i). In this case, by Lemma 3.12 below, the group $G$ contains an element of infinite order. The subgroup $H$ generated by an element $v$ of infinite order must have infinite index, since $G$ has one end and a group with an infinite cyclic subgroup of finite index has two ends (see [21, Corollary 11.34]). Thus, choosing $S_{0}=\widetilde{S} \cup\{v\}$ yields an infinite collection of disjoint rays in Cay $\left(G, S_{0}\right),\left\{\left\{v g_{i}, v^{2} g_{i}, v^{3} g_{i}, \ldots\right\}: i \in \mathbb{N}\right\}$, where $\left\{g_{i}: i \in \mathbb{N}\right\}$ are right coset representatives of $G / H$.

Lemma 3.12. Let $G$ be a one-ended group with finite generating set $\widetilde{S}$. Assume that there exists an $m \in \mathbb{N}$ such that for each $R \in \mathbb{N}$ there is a set $C_{R}$, consisting of $m$ vertices of length at least $R$ in $\operatorname{Cay}(G, \widetilde{S})$, such that the removal of $C_{R}$ disconnects 1 from "infinity". Then the group $G$ contains an element of infinite order.

Proof. Notice that the order of an element $z$ in $G$ for which the sequence $\left\{d_{G}\left(z^{n}, 1\right)\right\}_{n=1}^{\infty}$ is unbounded must be infinite. We use some ideas from the proof of the result on groups of linear growth in [16, 29] (see also [15, 20]) to produce such a $z$.

Assume that $\widetilde{S}$ is symmetric. Fix an order on the elements of $\widetilde{S}$ and then order all words of the same length in $\widetilde{S}$ using a lexicographic order. For each element $g$ of $G$, we call the first word in this order among the shortest words representing $g$ a distinguished word. It is easy to see that a subword of a distinguished word is distinguished. 
Let $w_{1}, \ldots, w_{m}$ be the distinguished words representing elements of the set $C_{R}$ for $R=m$. Let $u_{1}, \ldots, u_{m}$ be the starting pieces of length $m$ of $w_{1}, \ldots, w_{m}$. By taking a pointwise limit of distinguished words with indefinitely increasing lengths, we can find a geodesic ray $P$ starting at 1 in $G$ such that each finite piece of it is a distinguished word. Because 1 and "infinity" are disconnected in Cay $(G, \widetilde{S}) \backslash C_{R}, P$ must pass through $C_{R}$. Furthermore, thinking of $P$ as an infinitely long distinguished word, each subword of $P$ of length $m$ is one of the words $u_{1}, \ldots, u_{m}$. Thus, $P$ has at most $m$ distinct subwords of length $m$. Therefore, by [20, Theorem 3.3], each subword $w$ of $P$ of length at least $2 m$ is of the form $w=u t v$, where $t$ is $p$-periodic (i.e., $t=z^{k} r$, where $k$ is some exponent, $z$ is a word of length $p$ and $r$ is a word of length at most $p$ ) for some $0<p \leq m$, and $u$ and $v$ have lengths at most $m-p$. Apply this result to each of the initial subwords $w$ of $P$ of length at least $2 m$. It is clear that, when written in the form $w=u t v=u z^{k} r v$, infinitely many of the $w$ will have the same $z$. Since the lengths of all of the $u$ 's, $v$ 's, and $r$ 's are bounded above by $m$, it follows that the sequence $\left\{d_{G}\left(z^{n}, 1\right)\right\}_{n=1}^{\infty}$ is unbounded.

Remark 3.13. Recall that an infinite finitely generated group has one, two, or infinitely many ends (see [5, Theorem 8.32, p. 146]). Furthermore, a group with two ends contain $\mathbb{Z}$ as a subgroup of finite index. Hence, the question of whether or not a given group has a finite generating set for which the corresponding Cayley graph is not minor excluded is answered for one-ended groups and two-ended groups in Sections 3.4 and 3.2, respectively. The only case that remains open is the case of infinitely many ends.

\section{Open problems}

The following two problems are, in our opinion, the most intriguing open problems related to this paper.

Problem 4.1. Let $G$ be a group of asymptotic dimension at least 3. Does it follow that Cay $(G, S)$ is not minor excluded for any choice of generating set $S$ ?

Problem 4.2. Let $G$ be a group that is not virtually free. Does it follow that Cay $(G, S)$ is not minor excluded for some choice of generating set $S$ ?

\section{References}

[1] R. Aharoni, Menger's theorem for countable graphs. J. Combin. Theory Ser. B 43 (1987), no. $3,303-313$.

[2] G. N. Arzhantseva, P.-A. Cherix, On the Cayley graph of a generic finitely presented group. Bull. Belg. Math. Soc. Simon Stevin 11 (2004), no. 4, 589-601.

[3] P. Assouad, Sur la distance de Nagata, C. R. Acad. Sci. Paris Sér. I Math. 294 (1982), no.1, $31-34$.

[4] L. Babai, Some applications of graph contractions, J. Graph Theory 1 (1977), 125-130.

[5] M. R. Bridson, A. Haefliger, Metric Spaces of Non-Positive Curvature. Grundlehren der Mathematischen Wissenschaften, 319. Springer-Verlag, Berlin, 1999. 
[6] S. Buyalo, V. Schroeder, Elements of Asymptotic Geometry. EMS Monographs in Mathematics. European Mathematical Society (EMS), Zürich, 2007.

[7] R. Diestel, Graph Theory. Second edition. Graduate Texts in Mathematics, 173. SpringerVerlag, Berlin, 2000.

[8] G. A. Dirac, S. Schuster, A theorem of Kuratowski, Indagationes Math. 16 (1954), 343-348.

[9] C. Droms, Infinite-ended groups with planar Cayley graphs. J. Group Theory 9 (2006), no. 4, 487-496.

[10] C. Droms, B. Servatius, H. Servatius, Connectivity and planarity of Cayley graphs. Beiträge Algebra Geom. 39 (1998), no. 2, 269-282.

[11] J. Fakcharoenphol, K. Talwar, An improved decomposition theorem for graphs excluding a fixed minor, in: Approximation, randomization, and combinatorial optimization, 36-46, Lecture Notes in Comput. Sci., 2764, Springer, Berlin, 2003.

[12] A. Georgakopoulos, Characterising planar Cayley graphs and Cayley complexes in terms of group presentations. European J. Combin. 36 (2014), 282-293.

[13] M. Gromov, Asymptotic invariants of infinite groups, in: A. Niblo, M. Roller (Eds.) Geometric group theory, London Math. Soc. Lecture Notes, 182, 1-295, Cambridge University Press, 1993.

[14] R. Halin, A note on Menger's theorem for infinite locally finite graphs. Abh. Math. Sem. Univ. Hamburg, 40 (1974), 111-114.

[15] W. Imrich, N. Seifter, A bound for groups of linear growth. Arch. Math. (Basel) 48 (1987), no. 2, 100-104.

[16] J. Justin, Groupes et semi-groupes à croissance linéaire. C. R. Acad. Sci. Paris Sér. A-B 273 1971 A212-A214.

[17] P. Klein, S. Plotkin, S. Rao, Excluded minors, network decomposition, and multicommodity flow. In: Proc. 25th Annual ACM Symposium on the Theory of Computing, pp. 682-690, 1993.

[18] U. Lang, T. Schlichenmaier, Nagata dimension, quasisymmetric embeddings, and Lipschitz extensions. Int. Math. Res. Not. 2005, no. 58, 3625-3655.

[19] H. Levinson, Planar Cayley diagrams: accumulation points. Congr. Numer. 36 (1982), 207215.

[20] A. Mann, How Groups Grow. London Mathematical Society Lecture Note Series, 395. Cambridge University Press, Cambridge, 2012.

[21] J. Meier, Groups, Graphs and Trees. An Introduction to the Geometry of Infinite Groups, London Mathematical Society Student Texts, 73, Cambridge University Press, Cambridge, 2008.

[22] A. Naor, L. Silberman, Poincaré inequalities, embeddings, and wild groups. Compos. Math. 147 (2011), no. 5, 1546-1572.

[23] P. W. Nowak, G. Yu, Large Scale Geometry. EMS Textbooks in Mathematics. European Mathematical Society (EMS), Zürich, 2012.

[24] M. I. Ostrovskii, Expansion properties of metric spaces not admitting a coarse embedding into a Hilbert space, C. R. Acad. Bulgare Sci., 62 (2009), 415-420; Expanded version: arXiv:0903.0607. 
[25] M. I. Ostrovskii, Metric Embeddings: Bilipschitz and Coarse Embeddings into Banach Spaces. de Gruyter Studies in Mathematics, 49. Walter de Gruyter \& Co., Berlin, 2013.

[26] S. Rao, Small distortion and volume preserving embeddings for planar and Euclidean metrics. Proceedings of the Fifteenth Annual Symposium on Computational Geometry (Miami Beach, FL, 1999), 300-306, ACM, New York, 1999.

[27] D. Renault, Enumerating planar locally finite Cayley graphs. Geom. Dedicata 112 (2005), 25-49.

[28] D. J.S. Robinson, A Course in the Theory of Groups. Second edition. Graduate Texts in Mathematics, 80. Springer-Verlag, New York, 1996.

[29] A. J. Wilkie, L. van den Dries, An effective bound for groups of linear growth. Arch. Math. (Basel) 42 (1984), no. 5, 391-396.

[30] H. Zieschang, E. Vogt, H.-D. Coldewey, Surfaces and Planar Discontinuous Groups. Translated from the German by John Stillwell. Lecture Notes in Mathematics, 835. Springer, Berlin, 1980. 